\title{
Anterior two-thirds corpus callosotomy via stereotactic laser ablation
}

\author{
Michael Karsy, MD, PhD, Daxa M. Patel, MD, Kyle Halvorson, MD, Vance Mortimer, AS, \\ and Robert J. Bollo, MD
}

Department of Neurosurgery, Division of Pediatric Neurosurgery, University of Utah School of Medicine, and Primary Children's Hospital, Salt Lake City, Utah

\begin{abstract}
Anterior two-thirds corpus callosotomy is a common palliative surgical intervention most commonly employed in patients with atonic or drop seizures. Recently, stereotactic laser ablation of the corpus callosum without a craniotomy has shown promise in achieving similar outcomes with fewer side effects and shorter hospitalizations. The authors demonstrate ablation of the anterior two-thirds corpus callosum in a patient with Lennox-Gastaut syndrome and drug-resistant drop seizures. Technical nuances of laser ablation with 3 laser fibers are described. Postoperatively, the patient showed a significant reduction in seizure frequency and severity over a 9-month follow-up period.
\end{abstract}

The video can be found here: https://youtu.be/3-mMq5-PLiM.

KEYWORDS LITT; laser interstitial thermal therapy; corpus callosotomy; Lennox-Gastaut syndrome; drop attacks; epilepsy 